\title{
Ikaros family zinc finger 1 regulates dendritic cell development and function in humans
}

\author{
Urszula Cytlak (10 1, Anastasia Resteu1, Delfien Bogaert 2,3,4,5,6, Hye Sun Kuehn7, Thomas Altmann 1,8, \\ Andrew Gennery ${ }^{1,8}$, Graham Jackson ${ }^{8,9}$, Attila Kumanovics ${ }^{10}$, Karl V. Voelkerding ${ }^{10}$, Seraina Prader ${ }^{11}$, \\ Melissa Dullaers (1) 2,5,12, Janine Reichenbach ${ }^{11,13,14,15}$, Harry Hill ${ }^{10,16}$, Filomeen Haerynck ${ }^{2,3,5}$, \\ Sergio D. Rosenzweig ${ }^{7}$, Matthew Collin ${ }^{1,8}$ \& Venetia Bigley (1) ${ }^{1,8}$
}

Ikaros family zinc finger 1 (IKZF1) is a haematopoietic transcription factor required for mammalian B-cell development. IKZF1 deficiency also reduces plasmacytoid dendritic cell (pDC) numbers in mice, but its effects on human DC development are unknown. Here we show that heterozygous mutation of IKZFI in human decreases pDC numbers and expands conventional DC1 (cDC1). Lenalidomide, a drug that induces proteosomal degradation of IKZF1, also decreases pDC numbers in vivo, and reduces the ratio of pDC/cDC1 differentiated from progenitor cells in vitro in a dose-dependent manner. In addition, non-classical monocytes are reduced by IKZF1 deficiency in vivo. DC and monocytes from patients with IKZF1 deficiency or lenalidomide-treated cultures secrete less IFN- $\alpha, T N F$ and IL-12. These results indicate that human DC development and function are regulated by IKZF1, providing further insights into the consequences of IKZF1 mutation on immune function and the mechanism of immunomodulation by lenalidomide.

\footnotetext{
${ }^{1}$ Institute of Cellular Medicine, Newcastle University, Framlington Place, Newcastle upon Tyne NE2 4HH, UK. ${ }^{2}$ Primary Immunodeficiency Research Lab, Department of Pulmonary Medicine, Ghent University Hospital, C. Heymanslaan 10, 9000 Ghent Belgium. ${ }^{3}$ Department of Paediatrics Division of Paediatric Immunology and Pulmonology, Ghent University Hospital, C. Heymanslaan 10, 9000 Ghent Belgium. ${ }^{4}$ Center for Medical Genetics, Ghent University Hospital, C. Heymanslaan 10, 9000 Ghent Belgium. ${ }^{5}$ Center for Primary Immunodeficiency Jeffrey Modell Diagnosis and Research Centre, Ghent University Hospital, Heymanslaan 10, 9000 Ghent Belgium. ${ }^{6}$ Laboratory of Immunoregulation, VIB Inflammation Research Center, Technologiepark 927,9052 Ghent Belgium. ${ }^{7}$ Department of Laboratory Medicine NIH Clinical Center, National Institutes of Health, 10 Center Drive-MSC 1508, Bethesda, MD 20892-1508, USA. ${ }^{8}$ Newcastle upon Tyne Hospitals NHS Foundation Trust, Freeman Road, Newcastle upon Tyne NE7 7DN, UK. ${ }^{9}$ Northern Institute for Cancer Research, Newcastle University, Paul O'Gorman Building, Framlington Place, Newcastle upon Tyne NE2 4AD, UK. ${ }^{10}$ Department of Pathology, University of Utah, 50 North Medical Drive, Salt Lake City, UT 84132, USA. ${ }^{11}$ Division of Immunology, University Children's Hospital Zurich, Steinwiesstrasse 75, 8032 Zurich Switzerland. ${ }^{12}$ Department of Internal Medicine, Ghent University, De Pintelaan 185, 9000 Ghent Belgium. ${ }^{13}$ Children's Research Centre, University Children's Hospital Zurich, Steinwiesstrasse 75, 8032 Zurich Switzerland. ${ }^{14}$ Institute for Regenerative Medicine (IREM) Associated Group, University of Zurich, Wagistrasse 12, 8952 Schlieren, Zurich, Switzerland. ${ }^{15}$ Competence Center for Applied Biotechnology and Molecular Medicine (CABMM), University of Zurich, Irchel Campus, Winterthurerstr. 190, 8057 Zurich Switzerland. ${ }^{16}$ Department of Paediatrics and Medicine, University of Utah, 50 North Medical Drive, Salt Lake City, UT 84132, USA. These authors contributed equally: Matthew Collin, Venetia Bigley. Correspondence and requests for materials should be addressed to V.B. (email: venetia.bigley@ncl.ac.uk)
} 
ffective immunity requires the coordinated development and response of immune cells. This process is orchestrated by transcription factors (TFs), which may act in multiple lineages and govern the expression of both differentiation and functional gene sets. The in vivo functions of specific TFs may be interrogated through the study of primary immunodeficiencies resulting from germline mutations, an approach which offers a wealth of biological insights ${ }^{1,2}$.

Dendritic cells (DCs) initiate tolerance or immunity through presentation of antigen and stimulation of naive $T$ cells ${ }^{3}$. In addition, they regulate a range of leukocyte responses including B-cell survival ${ }^{4}$ and class switching ${ }^{5}$, natural killer cell proliferation and homeostasis ${ }^{6}$ and monocyte and neutrophil chemotaxis ${ }^{7}$. DCs consist of two main subsets, plasmacytoid DCs (pDCs) and myeloid or conventional DCs (cDCs), each associated with specific functions ${ }^{8}$. Human $\mathrm{pDCs}$ express CD123/IL-3R, CD303/BDCA-2 and CD304/BDCA-4 and, in common with pDCs of all species, secrete large amounts of interferon- $\alpha$ (IFN- $\alpha$ ) in response to viruses and other pathogens 9 . Two subsets of cDCs are described; $c D C 1$ and $c D C 2$. In humans these are differentiated by the expression of CD141 and CLEC9A (cDC1) or $\mathrm{CD} 1 \mathrm{c}(\mathrm{cDC} 2)$. $\mathrm{cDC} 1$ are specialised in antigen cross-presentation to $\mathrm{CD}^{+} \mathrm{T}$ cells, $\mathrm{T}$ helper type 1 polarisation of $\mathrm{CD} 4^{+} \mathrm{T}$ cells and type III IFN production ${ }^{10}$. Human $\mathrm{cDC} 2 \mathrm{~s}$ are the predominant interleukin-12 (IL-12) secretors, showing plasticity in T-cell polarisation depending on the environmental stimuli ${ }^{11}$.

pDCs and cDCs develop independently of monocytes under the control of specific TFs, largely mapped through the analysis of knockout mice ${ }^{2}$. PU.1 and GATA2 are required for specification of all DCs ${ }^{13}$, pDCs are dependent upon IRF8 and E2.2 ${ }^{14}, \mathrm{cDC} 1$ on IRF8, Id2 and BATF3 ${ }^{15-17}$ and $\mathrm{CDC} 2$ on IRF4 ${ }^{18}$. Classical monocytes, expressing CD14 in human (Ly6C in mouse), require KLF4 at the progenitor stage ${ }^{19}$. Non-classical monocytes express $\mathrm{CD} 16$ and can arise from conversion of $\mathrm{CD} 14^{+}$monocytes in the periphery $^{20}$.

Ikaros family zinc finger 1 (IKZF1) is a zinc finger TF and member of the IKAROS gene family, with prominent roles in lymphocyte development and proliferative responses ${ }^{21}$. Mutation of $I k z f 1$ has also been shown to have a dose-dependent effect upon DC development in the mouse. Homozygous $I k z f 1^{\mathrm{L} / \mathrm{L}}$ mice, expressing low levels of wild-type Ikzf1, have a specific defect of pDCs and loss of IFN- $\alpha$ production ${ }^{22}$. The null allele $\left(I k z f 1^{\mathrm{C} / \mathrm{C}}\right)$ prevents formation of $\mathrm{pDCs}$ and $\mathrm{cDC} 2 \mathrm{~s}$, maintaining a reduced population of $\mathrm{cDCl}$, whereas the dominant negative DNA binding domain mutant (Ikzf1 $\left.{ }^{\mathrm{DN} / \mathrm{DN}}\right)$ lacks all $\mathrm{DCs}^{23}$. Together, these results indicate that murine $\mathrm{pDCs}$ are most sensitive to Ikzf1 deficiency and $\mathrm{cDC} 1 \mathrm{~s}$ the least.

The importance of IKZF1 in human biology is illustrated by its pathogenic involvement in autoimmune disease (systemic lupus erythematosus) and haematopoietic malignancies (B-cell acute lymphoblastic leukaemia), including blastic plasmacytoid dendritic cell neoplasms (BPDCNs), characterised by the expression of pDC markers and CD56 on malignant cells ${ }^{24}$.

Human germline heterozygous IKZF1 mutations, resulting in haploinsufficiency, cause a variably penetrant combined immunodeficiency associated with progressive attrition of $\mathrm{B}$ cells, hypogammaglobulinaemia and skewing of T-cell subsets ${ }^{25-27}$. Clinical manifestations include recurrent or severe respiratory tract infections, autoimmune phenomena and a predisposition to childhood B-cell acute lymphoblastic leukaemia.

IKZF1 is also known to be a key target of thalidomide and its derivatives, used to treat myeloma and $5 \mathrm{q}$-myelodysplasia. It has recently been shown that their therapeutic actions include activation of Cereblon-dependent ubiquitination and proteasomal degradation of IKZF1 and IKZF3 $3^{28,29}$. Thus, exposure to lenalidomide induces IKZF1 deficiency offering a further opportunity to manipulate IKZF1 levels in vivo or during differentiation and functional analysis of human cells in vitro.

Prompted by the knowledge that murine pDC development is dependent upon Ikzf1, here we investigate whether IKZF1 mutation or inhibition with lenalidomide causes pDC deficiency in humans, using phenotypic and functional analyses performed on patients with IKZF1 haploinsufficiency, those receiving lenalidomide, or on progenitor cell cultures exposed to lenalidomide in vitro. In addition to $\mathrm{pDC}$ deficiency, we observe a relative increase in $\mathrm{CDC} 1$ in vivo and in vitro and a loss of non-classical monocytes in vivo. In the presence of IKZF1 deficiency, pDCs produce less IFN- $\alpha$, pDCs and monocytes secrete less tumor necrosis factor (TNF), and $\mathrm{cDC} 1$, although increased, produce less IL-12. These results extend the known functions of IKZF1 to include the regulation of human DC haematopoiesis.

\section{Results}

IKZF1 haploinsufficiency cohort. The clinical features, mutations and B-cell phenotype of 20 individuals from 4 families with heterozygous IKZF1 mutations have been previously reported. Families B, C and F were studied by Kuehn et al. ${ }^{25}$. Members of family $G$ have been recently described ${ }^{27}$. Replicate B-cell counts performed on blood taken for this study were congruent with the analyses previously reported. These and further details are summarised in Supplementary Table 1.

pDC deficiency and $\mathrm{cDC} 1$ expansion in IKZF1 mutation. In order to map the global perturbation of DC haematopoiesis induced by $I K Z F 1$ mutation, an unsupervised phenotypic analysis and enumeration was initially conducted using a member of family B (B5) and a control. The FlowSOM algorithm was used to cluster, visualise and compare equal numbers of data from the Lineage ${ }^{-} \mathrm{HLA}-\mathrm{DR}^{+}\left(\mathrm{Lin}^{-} \mathrm{DR}^{+}\right) \mathrm{CD}^{-}$gate of a 16 -colour flow cytometry panel (Supplementary Figure 1a, Supplementary Table 2). This algorithm clusters cells of the same phenotype into populations, each represented as a node. Nodes are presented as coloured metaclusters on a minimal spanning tree, defining the relationships between nodes ${ }^{30}$ (Fig. 1a). With an equal number of events from the affected and unaffected individual, FlowSOM identified 6 metaclusters: 1 corresponding to $\mathrm{CD}_{123}{ }^{+} \mathrm{pDCs}$ (blue nodes), 1 corresponding to $\mathrm{CD} 141^{+} \mathrm{BTLA}^{+} \mathrm{cDC}^{\mathrm{s}}$ (turquoise nodes) and the remaining 4 corresponding to $\mathrm{CD} 11 \mathrm{c}^{+} \mathrm{CD} 1 \mathrm{c}^{+} \mathrm{CD} 2^{+} \mathrm{cDC} 2 \mathrm{~s}$ (red nodes). Within metaclusters it was possible to discern a $\mathrm{CD} 2^{+}$node of $\mathrm{pDCs}$, and variable expression of CD2, CD5 and CD1c forming discrete nodes of cDC2s. To analyse the differences between the affected and unaffected individuals, the relative contribution of cells from each individual to each node was assessed (Fig. 1b). IKZF1 mutation was associated with an overall relative reduction in $\mathrm{pDCs}$, but increase in cDC1s. There was an altered distribution of cells within the $\mathrm{cDC} 2$ nodes. The relative loss of pDCs was consistent with the highest expression of IKZF1 mRNA and protein in this subset of DCs (Fig. 1c, d, Supplementary Figure 1b). For comparison, IKZF3 expression is shown (Fig. 1c).

DC subset skewing in IKZF1 haploinsufficiency. The relative decrease in pDCs and expansion of myeloid cDC1s and cDC2s was further defined by performing absolute whole blood counts on all patients compared with healthy controls. Within the CD3 ${ }^{-}$ mononuclear cell gate, the $\mathrm{HLA}-\mathrm{DR}^{+} \mathrm{CD} 4^{+}$population contained $\mathrm{CD}_{14}{ }^{+}$classical and $\mathrm{CD}_{1} 6^{+}$non $^{-}$classical monocytes, $\mathrm{CD} 123^{+}$

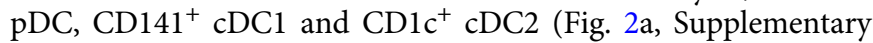
Figure 1c). All affected individuals had a profound reduction in pDCs but expansion in cDC1s $(p<0.05$, by two-tailed Mann-Whitney $U$-test) (Fig. 2b). In absolute counts, cDC2s 
a

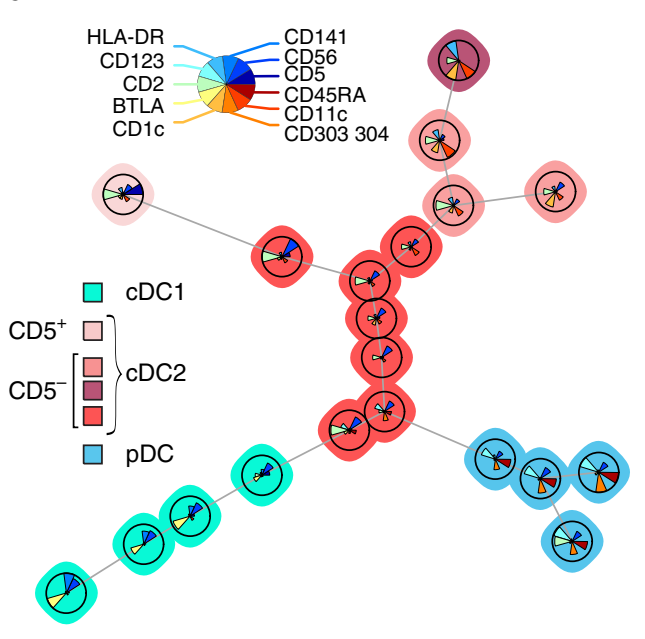

C

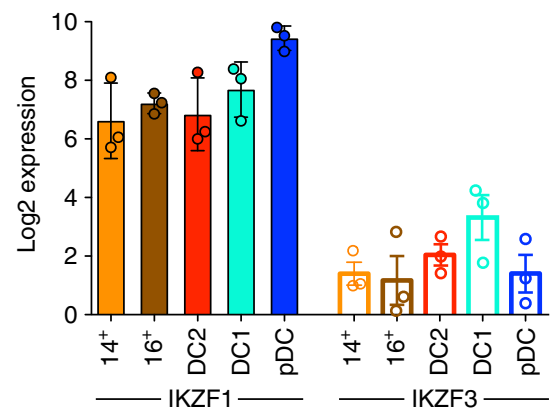

b

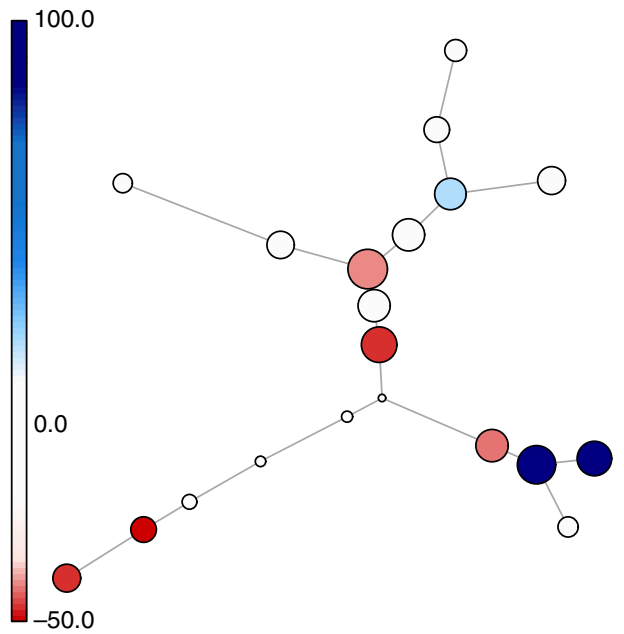

d

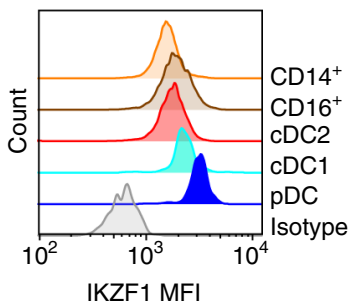

Fig. 1 FlowSOM analysis of PB reveals a reduced proportion of pDC but increased cDC1 in a patient carrying IKZF1 mutation. a Minimal spanning tree visualisation of a self-organising map using compensated flow cytometric data from a family B member (B5) compared to a travel control (equal number of events). Data were taken from the lineage (CD3, CD7, CD14, CD16, CD19, CD20)-negative HLA-DR-positive gate. FlowSOM nodes represent clusters of cells. Metaclusters of the nodes, determined by the map, are represented by the background colour of the nodes. Star charts represent the mean marker value of cells in that node with the height of each part corresponding to marker intensity. $\mathbf{b}$ Comparison between samples used to generate the map; size of nodes represents proportional number of cells in each node, colour represents proportional differences between samples with red and blue indicating higher or lower numbers in IKZF1 mutation compared to wild type, respectively. c mRNA and $\mathbf{d}$ protein expression of IKZF1 in healthy donor monocytes and dendritic cells by NanoString gene expression analysis of FACS sorted cells or intracellular flow cytometry, respectively ( $n=3$ donors for each experiment). IKZF3 mRNA expression is shown for comparison in c. cDC1/2, conventional dendritic cell 1/2; pDC, plasmacytoid dendritic cell; $14^{+}$CD14 ${ }^{+}$, classical monocyte; $16^{+} \mathrm{CD} 16^{+}$, non-classical monocyte

were not affected. Classical monocytes were also in the normal range but non-classical monocytes were reduced, even in patients who had received no therapy $(p<0.05$, by two-tailed Mann-Whitney $U$-test). Quantitative changes were less pronounced in family $\mathrm{F}$ carrying a multi-gene deletion on chromosome 7, encompassing IKZF1.

There was no effect of age on the DC phenotype, which was present in clinically asymptomatic and symptomatic individuals (Fig. 2c). An increased proportion of $\mathrm{CD} 56^{+}$cells was confirmed in all three DC subsets (Fig. 2d).

IKZF1 deficiency and pDC depletion in lenalidomide treatment. An independent verification of the effect of IKZF1 mutation on pDC development was sought through the analysis of patients receiving lenalidomide for haematological malignancy. Patient characteristics are summarised in Supplementary Table 3. Owing to the fact that a range of lenalidomide dosing schedules are employed, the level of IKZF1 protein was first quantified by intracellular flow cytometry of peripheral blood B cells. Lenalidomide treatment on the day of sample analysis resulted in a reduction in B-cell IKZF1 protein, comparable to that seen in heterozygous IKZF1 mutation in family G (Fig. 3a). Patients on maintenance lenalidomide sampled between treatment courses had intermediate levels that correlated with dose (Fig. 3b). A negative correlation between lenalidomide dose and the number of circulating B cells was also observed. (Fig. 3c).

Absolute pDC counts showed a significant positive correlation with IKZF1 protein level in 24 patients treated with lenalidomide $(n=22)$ or pomalidomide $(n=2)$ by linear regression analysis $\left(r^{2}=0.6561, p<0.0001\right)$. The inclusion of 4 healthy controls and 3 affected family $\mathrm{G}$ members did not significantly alter the slope or significance of the linear regression analysis $\left(r^{2}=0.6541\right.$, $p<0.0001$ ) (Fig. 3d).

Patients on lenalidomide also showed a reduction in $\mathrm{CD}^{+} 6^{+}$ non-classical monocytes, reaching statistical significance $(p=0.02$ by Mann-Whitney $U$-test) in those with the lowest IKZF1 protein levels (defined as R2 in Fig. 3d). Unlike the families with IKZF1 mutation, lenalidomide treatment was associated with a slight depression in $\mathrm{CDC} 2$ and no increase in $\mathrm{cDC} 1$ (Fig. 3e) compared to healthy controls.

Lenalidomide also causes depletion of IKZF3, but IKZF1 is expressed at more than 100 times the level of IKZF3 in human pDCs (mean $\log 29.8$ and 1.4 respectively; Fig. 1c). 
a

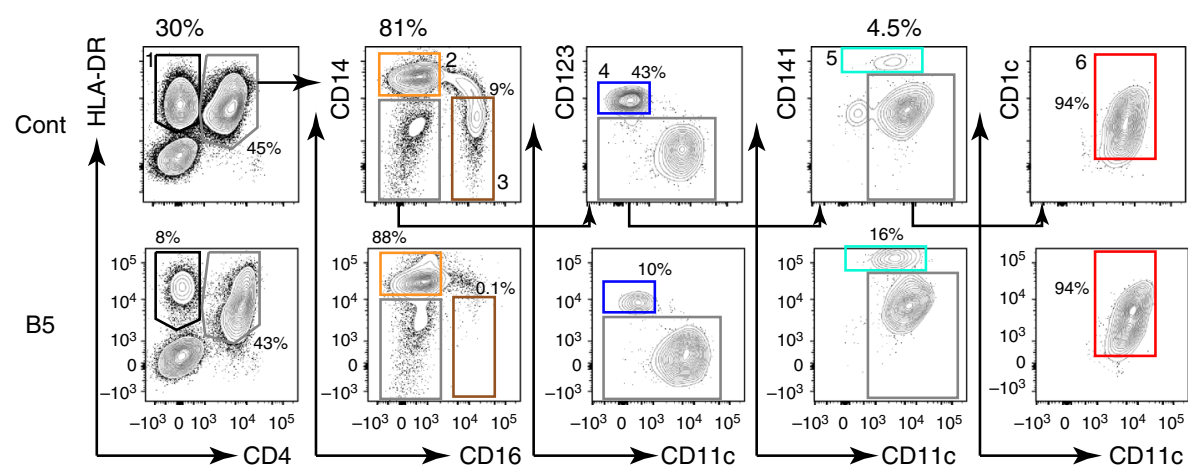

b
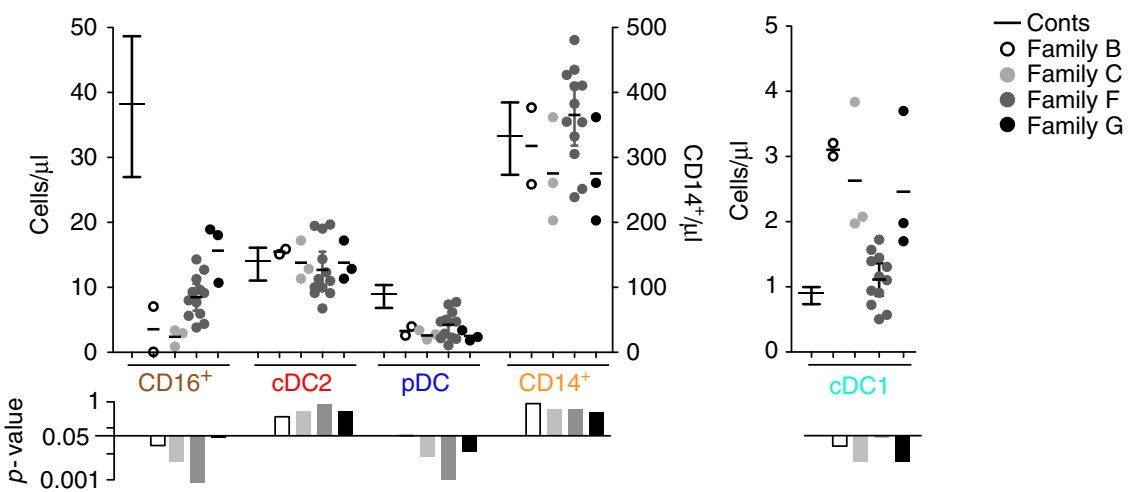

C
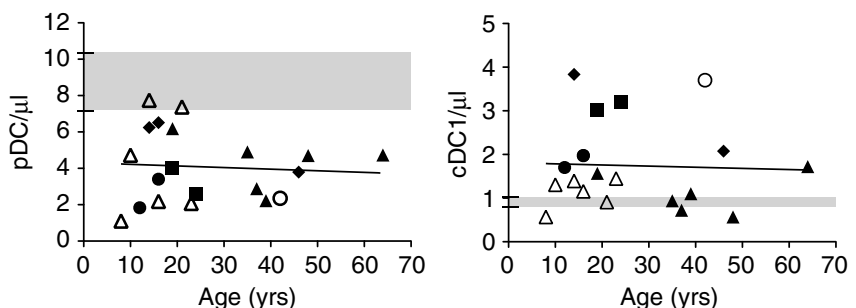

d
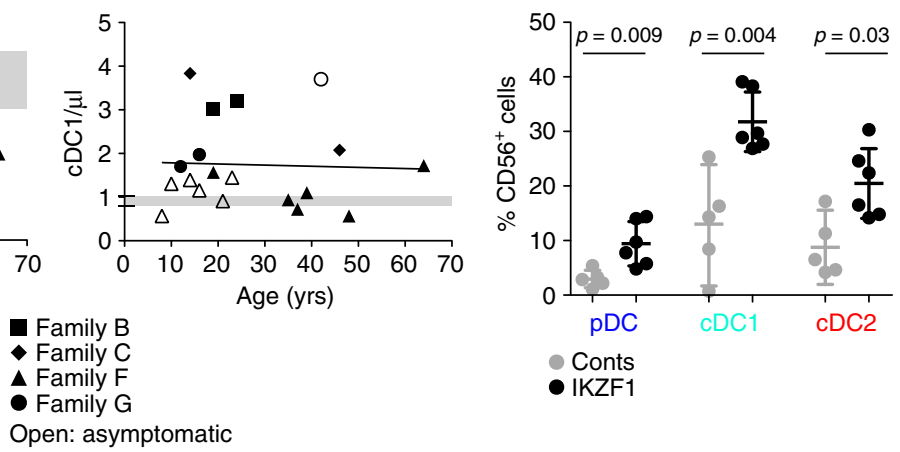

Fig. 2 Reduced $p D C$ and non-classical monocytes but expanded $C D C 1$ in the IKZF1 haploinsufficiency cohort. a Flow cytometric quantification of PB monocyte and dendritic cell (DC) subsets showing HLA-DR ${ }^{+} \mathrm{CD} 4^{-}$B cells, CD14 ${ }^{+}$classical (orange) and CD16 ${ }^{+}$non-classical monocytes (brown), CD123 ${ }^{+}$ plasmacytoid DCs (pDC) (blue), CD141 ${ }^{+}$conventional DC1 (cDC1) (turquoise) and CD1 ${ }^{+} \mathrm{CDC} 2$ (red) in healthy donor (cont, (control)) and representative individual with IKZF1 mutation. b Absolute counts of monocytes and DCs in 20 individuals from 4 affected families compared to $n=32$ healthy donors. Bars show mean $\pm 95 \%$ confidence interval $(\mathrm{Cl}$ ). Histograms show subset-specific $p$-values for each family compared to healthy donors by two-tailed Mann-Whitney $U$-test with significance set at $p<0.05$. c Absolute pDC or cDC1 counts plotted against the age of the individual. Grey zones represent the normal range of healthy controls $(n=32)$, black lines represent linear regression analysis with $p=0.77$ and $p=0.86$, respectively. $\mathbf{d}$ Proportion of cells expressing CD56 in healthy donors $(n=5)$ and individuals carrying IKZF1 mutation $(n=6)$. Bars represent mean $\pm 95 \% \mathrm{Cl}$. The $p$-values derived from twotailed Mann-Whitney U-test

Reduced IL-12 and IFN- $\alpha$ production in IKZF1 deficiency. Functional defects associated with loss of IKZF1 were investigated by examining intracellular cytokine production by specific DC and monocyte subsets in response to a cocktail of Toll-like receptor (TLR) agonists (polyinosinic:polycytidylic acid (poly(I: C)), lipopolysaccharide (LPS), CL075 and CpG). In healthy control peripheral blood mononuclear cells (PBMCs), no differences were observed in cell-specific production of IFN- $\alpha$, IL-12 or TNF in response to the relevant single TLR agonist compared to the cocktail (Supplementary Figure 2a).

Individuals with IKZF1 mutation and healthy control PBMCs with or without exposure to lenalidomide were examined. TNF production by all DC and monocyte subsets was reduced in the presence of IKZF1 mutation or exposure to lenalidomide (Fig. 4a, b, Supplementary Figure $2 \mathrm{~b}, \mathrm{c}$ ). IFN- $\alpha$ production by $\mathrm{pDC}$ and monocytes was also abolished or greatly reduced, respectively, on a per-cell basis, especially with IKZF1 mutation. Production of IL-12 by myeloid $\mathrm{CDC} 1 \mathrm{~s}$ and $\mathrm{CDC} 2 \mathrm{~s}$ was also reduced in both conditions. IL-10 was quite strongly induced by lenalidomide in monocytes and myeloid cells but was inversely affected by IKZF1 mutation.

In an attempt to dissect whether the loss of IL-12 production was a secondary effect of the reduction in IFN- $\alpha$ secretion by pDCs, it was observed that production of IFN- $\alpha$ by healthy control pDCs could be abrogated by ligation of CD303 and CD304 with anti-CD303/4 antibodies. Although exogenous 
a

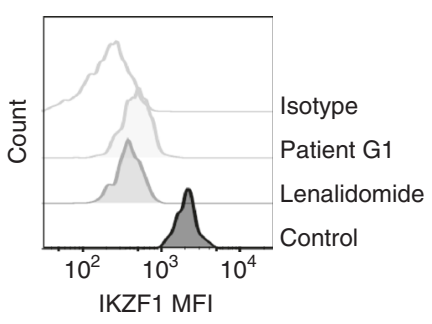

C

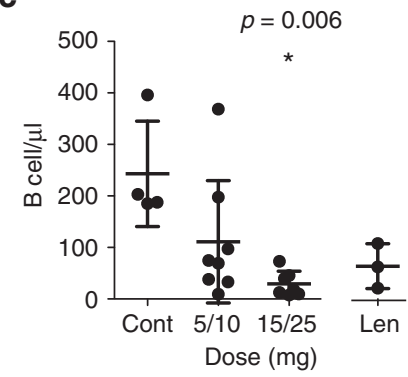

b

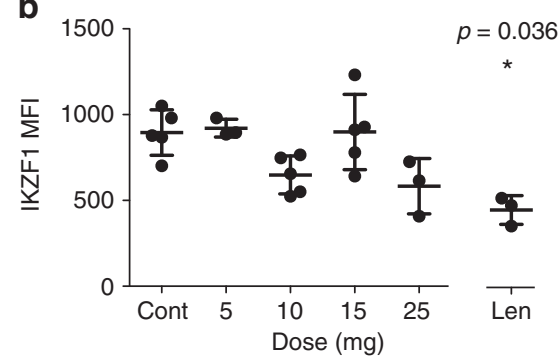

d

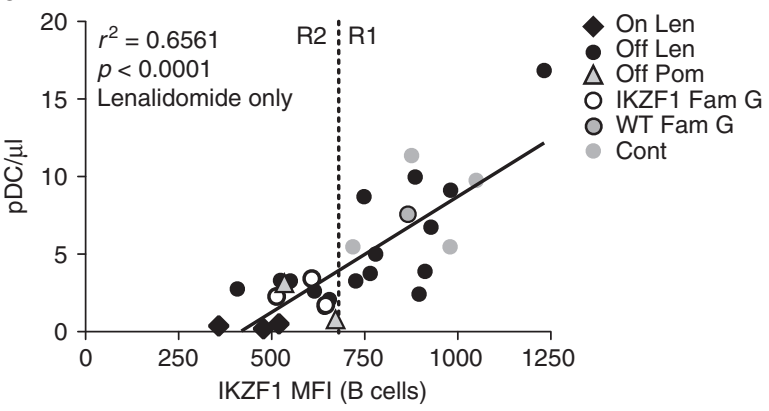

e

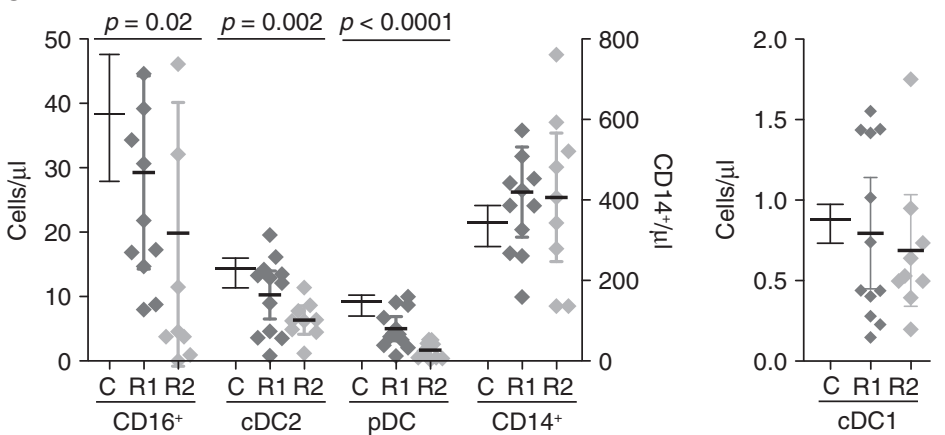

Fig. 3 Dose-dependent reduction of IKZF1 protein and pDCs in patients receiving lenalidomide treatment. a Intracellular flow cytometric quantification (mean fluorescence intensity (MFI)) of IKZF1 protein levels in B cells (identified as SSC ${ }^{10 w}$ Lin $^{-} \mathrm{DR}^{+} \mathrm{CD} 2^{-}$cells) of a family $\mathrm{G}$ member carrying IKZF1 mutation, patient on lenalidomide treatment, a healthy donor and isotype control. $\mathbf{b}$ Intracellular IKZF1 level (MFI) in healthy donors $(n=5)$, patients tested at the end of a week off treatment with specified dose of lenalidomide $(n=16)(5,10,15$ or $25 \mathrm{mg}$ daily) and patients taking lenalidomide (Len) on the day of analysis $(n=3)\left(15 \mathrm{mg}\right.$ daily). Bars represent mean \pm s.d. ${ }^{*} p=0.036$ by two-tailed Mann-Whitney U-test versus healthy donor controls. c Summary of absolute B-cell counts in whole blood from healthy donors $(n=4)($ Cont), patients tested at the end of a week off treatment with specified dose of lenalidomide $(n=16)(5$ or 10,15 or $25 \mathrm{mg}$ daily) and patients taking lenalidomide on the day of analysis $(n=3)(15 \mathrm{mg}$ daily). Bars represent mean \pm s.d. ${ }^{*} p=0.006$ Mann-Whitney U-test versus healthy donor controls. $\mathbf{d}$ IKZF1 protein levels (MFI) plotted against absolute plasmacytoid dendritic cell ( $\left.p D C\right)$ counts in affected $(n=3)$ and unaffected $(n=1)$ members of family $\mathrm{G}$ (white or grey open circles, respectively), patients after a week off lenalidomide $(n=22)$ (black circles) or taking lenalidomide on the day of analysis $(n=3)$ (black diamond) or healthy donors $(n=4)$ (grey circles). Data from two patients taking pomalidomide ( $4 \mathrm{mg}$ daily) are displayed (grey triangles). Black line represents linear regression analysis of lenalidomide-treated patients only. Inclusion of family $\mathrm{G}$ members and healthy controls in the linear regression analysis resulted in $r^{2}=0.6541, p<0.0001$. Inclusion of pomalidomidetreated patients resulted in $r^{2}=0.6419, p<0.0001$. Dashed line represents IKZF1 MFI $=680$ dividing group R1 (IKZF1 MFI $>680$ ) from group R2 (IKZF1 $\mathrm{MFI}<680$ ). e PB absolute monocyte and DC counts in healthy controls (C) compared to patients from group R1 and R2. The $p$-values from two-tailed Mann-Whitney U-test of R2 versus healthy donors

IFN- $\alpha$ had a slightly enhancing effect on the secretion of IL-12 by $\mathrm{cDC} 2$, the baseline production of IL-12 by $\mathrm{cDC} 2$ was not at all affected when IFN- $\alpha$ production by pDCs was completely blocked, suggesting that the loss of IL-12 production in the preceding experiments was directly attributable to loss of IKZF1 (Fig. 4c).

IKZF1 deficiency impairs pDC differentiation in vitro. The effect of IKZF1 deficiency on human DC development was examined in vitro. DC subsets were generated from human bone marrow $\mathrm{CD} 34^{+}$progenitors after 22 days (D22) of culture in the presence of a lenalidomide titration.
DC subsets were identified by their surface marker expression profile corresponding to blood counterparts: $\mathrm{CD} 11 \mathrm{c}^{+} \mathrm{CD} 14^{+}$ monocytes, CD141 ${ }^{+} \mathrm{CLEC} 9 \mathrm{~A}^{+} \mathrm{cDC} 1, \mathrm{CD} 11 \mathrm{c}^{+} \mathrm{CD} 1 \mathrm{c}^{+} \mathrm{cDC} 2$ and $\mathrm{CD} 303^{+} \mathrm{CD} 304^{+} \mathrm{CD} 123^{+}$pDC (Fig. 5a, Supplementary Figure 1d). There was a negative correlation between lenalidomide concentration in the culture and IKZF1 protein level in $\mathrm{Lin}^{-} \mathrm{DR}^{+}$cells at D22 of culture (Fig. 5b). This was associated with a reduction in pDC and $\mathrm{CDC} 2$ but increase in $\mathrm{CDC} 1$ output. $\mathrm{CD} 14^{+}$cells were unaffected (Fig. 5c). A reduction in the number of cells generated per input progenitor was seen at lenalidomide concentrations above the published in vivo plasma $C_{\max }$ for therapeutic dosing $(1.7-2.3 \mu \mathrm{M})^{31}$ (Fig. 5d), but there remained a clear dosedependent effect on the $\mathrm{cDC} 1 / \mathrm{pDC}$ ratio (Fig. 5e). 
a

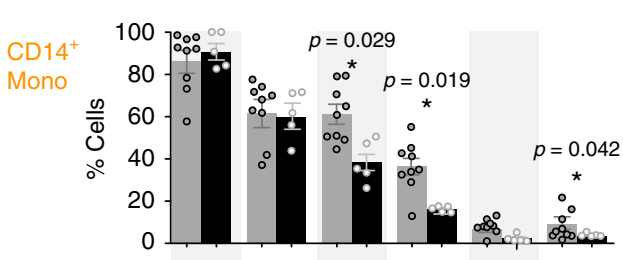

CD14
Mono

cDC2

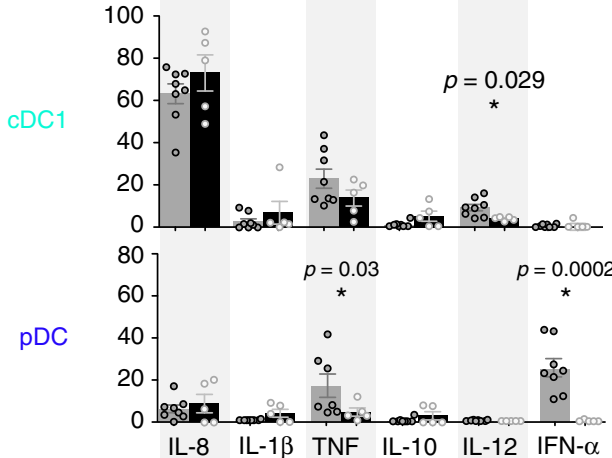

pDC
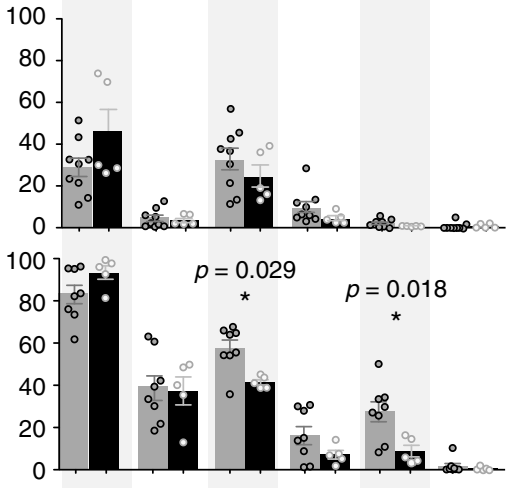

IL-8 IL-1 $\beta$ TNF IL-10 IL-12 IFN- $\alpha$

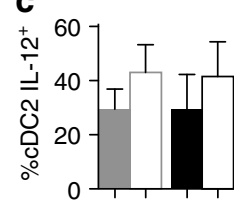

IFN- $\alpha-+-+$

$\alpha \mathrm{CD} 303 / 4-\ldots+$

TLR ++++ b
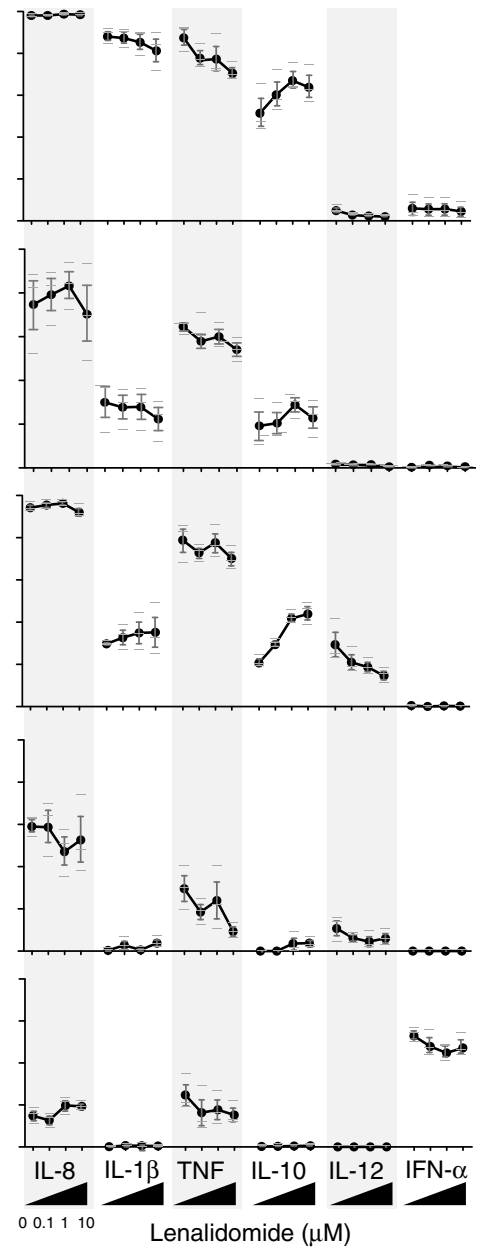

Fig. 4 Reduced production of TNF, IL-12 and IFN- $\alpha$ in IKZF1 deficiency. a Intracellular flow cytometric analysis of cytokine production (\% positive cells) in $\mathrm{PB} \mathrm{CD14^{+ }}$ monocytes (mono), CD14- monocytes and dendritic cell (DC) subsets (cDC2, cDC1, pDC) from $n=5$ individuals carrying IKZF1 mutation (black bars) or $n=8$ healthy donors (grey bars) following $14 \mathrm{~h}$ of stimulation of PBMCs with a cocktail of TLR agonists (CpG, poly(I:C), CLO75, LPS). Histograms represent mean and bars represent s.e.m. Dots represent individuals. The $p$-values derived from Mann-Whitney $U$-test of IKZF1 mutation versus healthy donors. $\mathbf{b}$ Analysis repeated on healthy donor PBMCs in the presence of increasing concentrations of lenalidomide $(0,0.1,1$ or $10 \mu \mathrm{M})$. Black dots represent mean, bars represent s.e.m. and lines represent individual data points from $n=3$ donors in each condition. $\mathbf{c}$ IL-12 or IFN- $\alpha$ production from cDC2 or pDC, respectively, from healthy donor PBMCs stimulated with TLR cocktail (TLR) and without (solid bars) or with (unfilled bars) the addition of exogenous IFN- $\alpha$ and without (grey) or with (black) anti-CD303 and anti-CD304 antibodies

\section{Discussion}

IKZF1 is a key regulator of haematopoiesis and a critical factor in murine lymphocyte development and function ${ }^{21}$. Normal IKZF1 protein levels are also necessary for the development of IFN- $\alpha$ producing $\mathrm{pDC}$ in mice 22,23 . Recent descriptions of human IKZF1 haploinsufficiency have confirmed its role in human lymphocyte biology but human DC development has not been studied $^{25-27}$. In this study we analysed blood monocytes and DCs from patients ex vivo carrying heterozygous IKZF1 mutations, or treated with lenalidomide, an IKZF1-depleting immunomodulatory drug. We also probed the effects of IKZF1 deficiency on human DC development and function in vitro.

In keeping with the pleiotropic actions of haematopoietic TFs, IKZF1 deficiency resulted in multi-lineage developmental and functional defects. In addition to the previously described progressive loss of $\mathrm{B}$ cells and skewing of T-cell subsets, we found deficiency of pDCs and non-classical monocytes but expansion of cDC1s. Classical monocytes and cDC2 remained numerically unaffected. The near universal finding of this antigen presenting cell phenotype, independent of age, lymphocyte phenotype or 
a

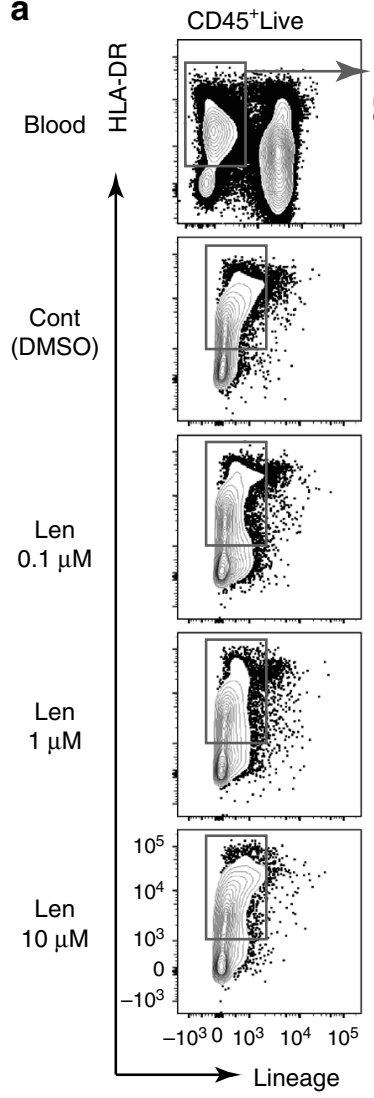

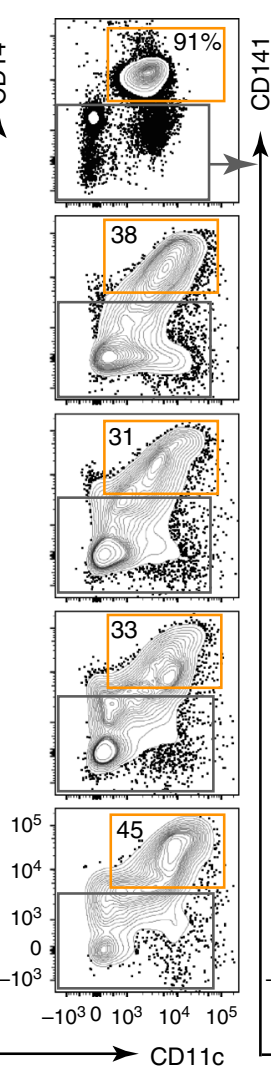

CD11c

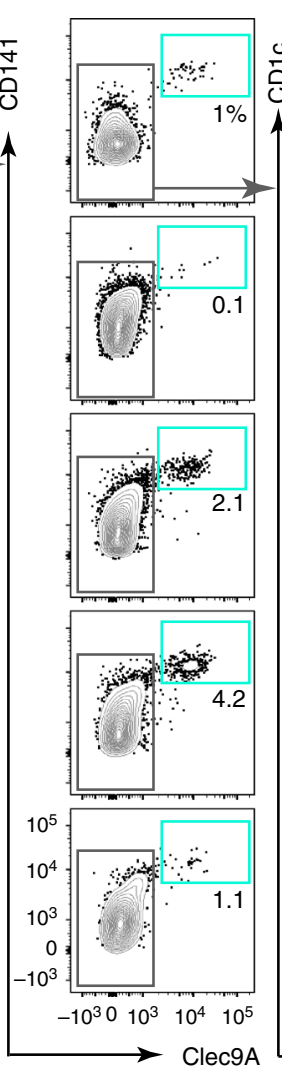

d

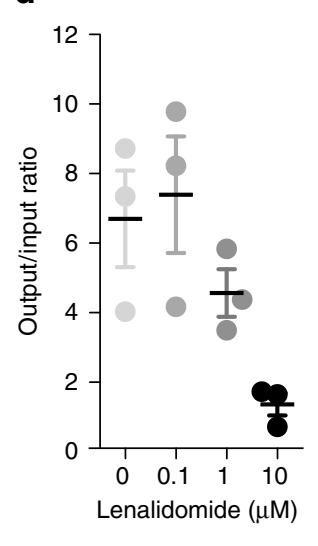

b

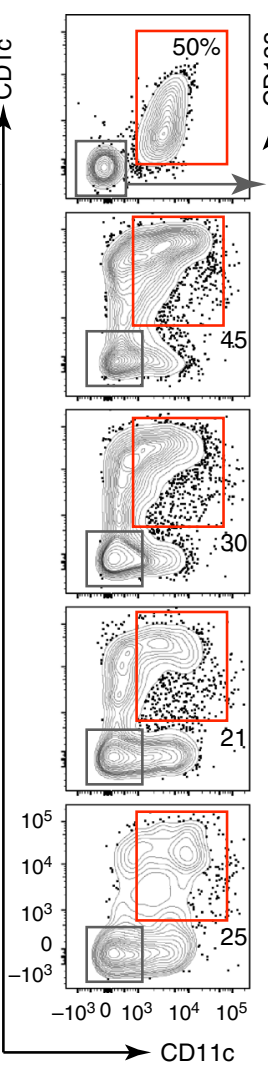

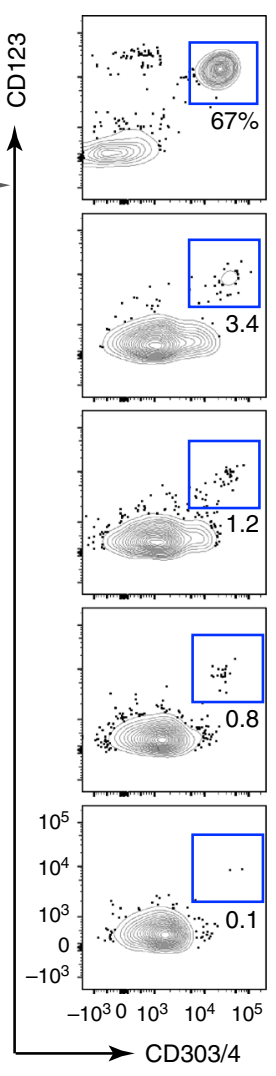

$\operatorname{Lin}^{-} \mathrm{DR}^{+}$

$(\mu \mathrm{M})$

0

0.1

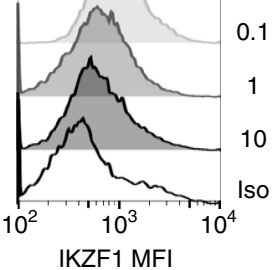

IKZF1 MFI

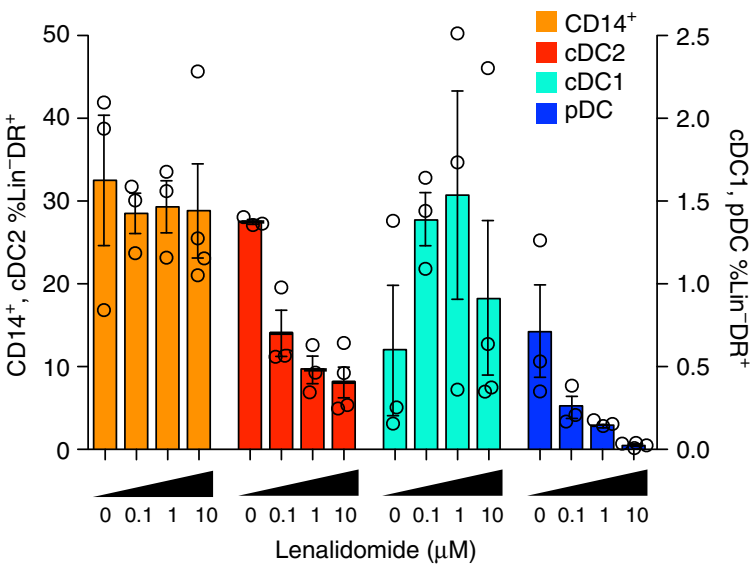

e

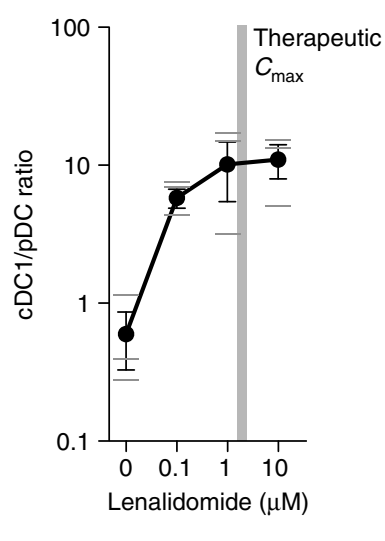

Fig. 5 Impaired in vitro pDC development in IKZF1 deficiency. a Phenotypic flow cytometric analysis of dendritic cell (DC) subsets generated after 22 days of culture in vitro, compared to PB counterparts. Found within the Lineage ${ }^{-} H L A-D R^{+}$gate were $C D 14^{+} C D 11 c^{+}$monocytes, CD141 ${ }^{+} C L E C 9 A^{+} \mathrm{cDC}$, $\mathrm{CD}_{11} \mathrm{c}^{+} \mathrm{CD} 1 \mathrm{c}^{+} \mathrm{CDC} 2 \mathrm{~s}$ and $\mathrm{CD} 123^{+} \mathrm{CD} 303^{+} \mathrm{CD} 304^{+} \mathrm{pDC}$. b IKZF1 protein levels, determined by flow cytometric analysis and IKZF1 mean fluorescence intensity (MFI), in $\mathrm{Lin}^{-} \mathrm{DR}^{+}$cells after 22 days of culture in the presence of a lenalidomide titration. c Summary of the proportion of in vitro generated Lin ${ }^{-} \mathrm{DR}^{+}$cells identified as CD14 ${ }^{+}$monocytes (orange), cDC2 (red), cDC1 (turquoise) and pDC (blue). Bars represent mean and s.e.m. of triplicate wells, dots represent single cultures. $\mathbf{d}$ Ratio of total number of $\mathrm{Lin}^{-} \mathrm{DR}^{+}$cells generated in vitro per input $\mathrm{CD} 34^{+}$cell in the presence of increasing concentrations of lenalidomide. e Lenalidomide dose response of the $\mathrm{CDC1}$ to $\mathrm{pDC}$ ratio of in vitro generated cells. Grey zone indicates published human in vivo plasma $C_{\text {max }}$ of lenalidomide $(450-600 \mathrm{ng} / \mathrm{ml}$ or $1.7-2.3 \mu \mathrm{M})$. Dots represent mean, bars represent s.e.m. and lines represent individual cultures

clinical status, provides a cellular signature of human IKZF1 mutation. The quantitative changes were remarkably similar in all individuals with missense proteins (families $\mathrm{B}$ and $\mathrm{C}$ ), or truncated protein (family $\mathrm{G}^{27}$ ), but less severe in members of family $\mathrm{F}$ who carry a heterozygous, 11-gene deletion of chromosome 7 . In homodimeric proteins, it has been proposed that a heterozygous missense mutation may result in a more severe phenotype than a null allele due to the lower proportion of WT/WT dimers $(25 \%$ versus $50 \%$, respectively) ${ }^{32}$. However, in the case of family $\mathrm{F}$, a compensatory effect due to the loss of additional genes cannot be excluded.

The requirement for IKZF1 in human pDC development and function mirrors that seen in the mouse and was supported by its high level of expression in healthy control pDCs. There was no significant increase in absolute number or proportion of $\mathrm{CDC} 1$ in mice carrying the heterozygous $I k z f 1 \mathrm{~L}$ allele, tested in cohorts of 3 animals $^{22}$, representing either a species or mutation-specific difference. Targets of IKZF1, identified by chromatin 
immunoprecipitation sequencing ${ }^{33}$, include ID2, suppression of which is necessary for pDC development and BATF3, required for $\mathrm{CDC} 1$ terminal differentiation. De-repression of these loci due to IKZF1 deficiency is consistent with the observed phenotype of absent pDCs but preserved or expanded cDC1s.

The reduction in non-classical monocytes, to our knowledge, has not been reported in Ikzf1-deficient mice. This finding was independent of therapeutic interventions including intravenous immunoglobulin and corticosteroid treatment, previously reported to result in transient depletion of $\mathrm{CD}^{+} 6^{+}$monocytes ${ }^{34}$. Ly6C $\mathrm{C}^{\text {low }}$ murine monocytes, corresponding to human $\mathrm{CD} 16^{+}$ non-classical monocytes, convert from classical monocytes under the control of NOTCH2 signalling stimulated by endothelial cell notch-ligand delta-like 1 (DLL1) ${ }^{35}$. The role of notch signalling in the generation of $\mathrm{CD}_{1} 6^{+}$classical monocytes is untested, but it is known that the regulation of notch target genes is IKZF1 dependent in human $\mathrm{T}_{\text {cells }}{ }^{36}$.

The cell-intrinsic effect of IKZF1 mutation on DC phenotype was confirmed in patients receiving therapeutic lenalidomide, known to target IKZF1 for proteosomal degradation ${ }^{28,29}$.

Varying lenalidomide dose schedules resulted in a range of IKZF1 levels in vivo, revealing a linear relationship between IKZF1 protein and the frequency of pDCs. Such an in vivo doseresponse effect would be difficult to demonstrate from the series of germline mutations that confer idiosyncratic, allele-specific effects upon protein structure and function. Parallel observations on the in vitro generation of DCs from primary bone marrow progenitors showed a lenalidomide dose-dependent decrease in the production of $\mathrm{pDCs}$ and increase in $\mathrm{cDC} 1 \mathrm{~s}$. Although the increased ratio of $\mathrm{cDC1}$ to $\mathrm{pDCs}$ was strikingly similar in the ex vivo analysis of patients with germline IKZF1 mutation and those treated with lenalidomide, $\mathrm{CDC} 1 \mathrm{~s}$ were not expanded and cDC2s were reduced by the drug. This may be due to the known myelosuppressive effect of lenalidomide as concentrations above the therapeutic $C_{\max }$ of lenalidomide resulted in a reduction in the cellular output per input progenitor cell in vitro. In addition, cereblon-dependent suppression of IRF4 by lenalidomide ${ }^{37}$ may contribute to the dose-dependent reduction in $\mathrm{CDC} 2$ seen in vivo and in vitro. While our data are unable to exclude an effect of IKZF3 deficiency on the DC phenotype in lenalidomide treatment, it is expressed at a much lower level than IKZF1 in human DCs and a role for this factor in DC differentiation has not been described in murine models.

In functional terms, IKZF1 haploinsufficiency resulted in perturbed cell-specific cytokine secretory responses to TLR agonists. Remaining pDCs were unable to secrete IFN- $\alpha$, production of IL12 by cDCs was reduced and all cells failed to elaborate as much TNF. A similar pattern was seen in healthy donor DCs exposed to lenalidomide. The reduction in IL-12 secretion contrasted with reports showing that lenalidomide does not compromise IL-12 production from monocyte-derived DCs (moDCs) stimulated with CD40L ${ }^{38,39}$. However, moDCs are not dependent on IKZF1 for development ${ }^{40}$ and in vitro stimulation with CD40L triggers IL-12 production through the non-canonical, nuclear factor (NF)-кB (p52/p100) pathway.

Our data are consistent with a direct effect of IKZF1 deficiency upon canonical NF- $\kappa \mathrm{B}$ (Rel-A/p50) signalling in which IKZF1 is necessary for the upregulation of Rel- $\mathrm{A}^{41}$ and is itself upregulated by LPS-TLR4 stimulation ${ }^{42}$. We considered the additional scenario that down regulation of IL-12 might have been an indirect effect of loss of type I IFN production by pDCs, as exogenous IFN augmented IL-12 production $^{43}$. However, CD303/CD304 ligation, which also abrogates IFN- $\alpha$, failed to reduce IL-12 production and lenalidomide resulted in a similar reduction in IL-12, despite only a modest fall in IFN- $\alpha$. From these observations we conclude that lower IL-12 production by cDCs was most likely intrinsic to loss of IKZF1.

The multi-lineage and multi-level influence exerted by haematopoietic TFs complicates the attribution of immunodeficiency resulting from $\mathrm{TF}$ mutation to defects in specific immune cell types. In DC deficiency states, the functional diversity of DCs, their combined roles in innate and adaptive immunity and their potential to both activate and tolerise add further complexity. In summarising the consequences of IKZF1 deficiency, pDC dysfunction is likely to play a role. An increased risk of bacterial infection, particularly respiratory infection in the context of germline haploinsufficiency, is consistent with the role of pDC in prompt bacterial clearance and limitation of inflammation in the lung ${ }^{44}$, in addition to their known anti-viral properties. Humoral immune responses are also dependent upon $\mathrm{pDC}$ function through the promotion of naive and memory B-cell proliferation, plasma cell differentiation and immunoglobulin secretion ${ }^{45}$. This is in keeping with a contribution of $\mathrm{pDC}$ deficiency to progressive hypogammaglobulinaemia seen in IKZF1 haploinsufficiency, despite the persistence of plasma cells in tissues ${ }^{25}$. In other settings, pDCs promote peripheral and central tolerance, through induction of natural and induced regulatory $\mathrm{T}$ cells and direct suppression of $\mathrm{T}$-cell responses ${ }^{46}$. Related to their tolerogenic role, pDCs in the bone marrow microenvironment have been shown to support multiple myeloma cell growth and mediate myeloma-associated immunodeficiency ${ }^{47}$. The loss of pDCs may therefore promote the development of autoimmunity in IKZF1 haploinsufficiency and confer therapeutic benefit in the treatment of multiple myeloma. These effects are potentially enhanced by an increase in the $\mathrm{cDC} 1 / \mathrm{pDC}$ ratio. $\mathrm{cDC} 1 \mathrm{~s}$, specialised for crosspresentation of antigen to cytotoxic $\mathrm{T}$ cells, are the most potent DCs in immunity to tumours and vaccinations. Consistent with this, in the murine model of multiple myeloma, lenalidomide synergistically enhances the anti-tumour effect of DC vaccines ${ }^{48}$ and in myeloma patients, lenalidomide enhances responses to a pneumococcal vaccine ${ }^{49}$

Finally, IKZF1 and pDC are connected in a number of other conditions. IKZF1 is a susceptibility locus in systemic lupus erythematosus, notable for a type I IFN signature and dysregulated pDC function (reviewed in ref. ${ }^{50}$ ). In BPDCN, frequently involving deletion or loss of function mutations of IKZF1, increased CD56 expression is a hallmark of the neoplastic pDC phenotype $^{24}$. In the studies described here, increased CD56 expression is seen to arise directly from IKZF1 deficiency.

In summary, our data demonstrate that in addition to its critical role in B-cell differentiation, IKZF1 is required for human pDC development and function. Together with the parallel expansion of $\mathrm{cDC} 1 \mathrm{~s}$ and reduction of non-classical monocytes, this comprehensively defines the cellular signature of IKZF1 haploinsufficiency. DC dysregulation is highly likely to have pathological consequences for immunity in germline IKZF1 mutation but confer additional therapeutic benefit in lenalidomide treatment of plasma cell dyscrasias. In common with other haematopoietic TFs, germline deficiency reveals multi-level and multi-lineage roles in immune cell development and function with effects in B-cell, T-cell, DC and monocyte lineages.

\section{Methods}

Study approval. The study was performed in accordance with the Declaration of Helsinki. Written informed consent was obtained from participants, or their parents, prior to recruitment. The study was approved by local review boards: NRES Committee North East-Newcastle and North Tyneside 1, 08/H0906/72; KEK-ZH Nr. 2015-0135; IRB 00029386; Ethical Committee of Ghent University Hospital, $2012 / 593$.

Patients. Individuals carrying an $I K Z F 1$ mutation, and family members, were recruited at their local medical centres in accordance with local ethical permissions 
All participating patients were included in the study. The family nomenclature (families B, C and F) corresponds to the nomenclature published in ref. ${ }^{25}$, with the exception of a newly described family ' $\mathrm{G}^{2749,50}$.

Patients receiving lenalidomide treatment were recruited from a local ambulatory myeloma clinic. There were no specific inclusion or exclusion criteria and analyses from all tested patients were included.

Flow cytometry and cell sorting. PBMCs, separated by density centrifugation, were stained in aliquots of $1-3 \times 10^{6}$ cells in $50 \mu \mathrm{l}$ of Dulbecco's phosphate-buffered saline with $2 \%$ fetal calf serum and $0.4 \%$ EDTA. Dead cells, usually $<5 \%$, were excluded by 4',6-diamidino-2-phenylindole (Partec) or Zombie (Biolegend). Analysis was performed with an LSRFortessa X-20 and sorting with a FACSAria III (BD Biosciences) running BD FACSDIVA ${ }^{\mathrm{TM}} 8.0 .1$ or 8.0 software, respectively. Data were processed with FlowJo 10.1r5 (Tree Star, Inc.). Absolute cell counts were obtained using TruCount ${ }^{\mathrm{TM}}$ tubes (BD Biosciences) with $200 \mu \mathrm{l}$ whole blood and $900 \mu \mathrm{l}$ of red cell lysis buffer. Intracellular staining was performed after surface staining, lysis and fixation (eBioscience) according to the manufacturer's instructions. Antibodies used are given in Supplementary Table 2.

FlowSOM analysis. Flow cytometric analysis of PBMCs from a member of family B (B5) carrying IKZF1 mutation and a travel control were analysed using an 18channel (16 fluorochromes, 2 light scatter) panel. Compensated FCS files were manually gated (FlowJo 10.1r5, Treestar, Inc.) to export $\mathrm{Lin}^{-} \mathrm{DR}^{+} \mathrm{CD} 14^{-}$cells.

FlowSOM 1.7.1 was used for further analysis. From each of the two files, 550 cells were randomly selected. A total of 12 surface markers were used for building the self-organising map (SOM): CD5, CD141, CD123, CD2, BTLA, HLA-DR, CD1c, CD303, CD304, CD11c, CD45RA, and BTLA. CD56 was included in visualisation.

SOM grid dimensions were set to $4 \times 5$ and the resulting SOM visualised in a minimal spanning tree with 20 nodes, corresponding to cell clusters. The maximum number of metaclusters, equivalent to predicted cell types, was set to 15 . The software identified 6 metaclusters, represented by background colour of the nodes.

The differences for each node were calculated by subtracting the number of patient cells from the number of healthy control cells.

DC functional analysis. The $3 \times 10^{6}$ PBMCs from healthy donors or individuals carrying heterozygous IKZF1 mutation were cultured in the presence poly(I:C) (10 $\mu \mathrm{g} / \mathrm{ml}$, Invivogen), LPS (5ng/ml, Sigma), CL075 (1 $\mu \mathrm{g} / \mathrm{ml}$, Invivogen) and CpG (ODN 2216, $7.5 \mu \mathrm{M}$, Invivogen) with or without IFN- $\alpha$ (3000 IU/ml, R\&D), with or without anti-CD303 and anti-CD304 (Biolegend), with or without $0.1,1$ or $10 \mu \mathrm{M}$ lenalidomide (Sigma). Cells were cultured for $14 \mathrm{~h}$ at $37^{\circ} \mathrm{C}, 5 \% \mathrm{CO}_{2}$, with addition of Brefaldin A $(10 \mu \mathrm{g} / \mathrm{ml}$, eBioscience) after $3 \mathrm{~h}$. For dead-cell exclusion (usually $<30 \%$ ) cells were stained with Zombie amine dye (Biolegend), surface markers and then intracellular cytokines antibodies after fixation and permeabilization (eBioscience), as above.

In vitro generation of DCs. CD34 ${ }^{+}$bone marrow progenitors were purified by fluorescence-activated cell sorting (FACS) (>98\% purity) and seeded (3000/well) onto OP9 stromal cells (5000/well) in 96-well U-bottomed plates. Cells were cultured in $200 \mu \mathrm{l} \alpha \mathrm{MEM}$ (Gibco ${ }^{\mathrm{TM}}$ ) supplemented with $1 \%$ penicillin/streptomycin (Sigma), $10 \%$ fetal calf serum (Gibco), $20 \mathrm{ng} / \mathrm{ml}$ granulocyte-macrophage colonystimulating factor (R\&D systems), $100 \mathrm{ng} / \mathrm{ml}$ Flt3-ligand (Immunotools), $20 \mathrm{ng} / \mathrm{ml}$ stem cell factor (Immunotools), with or without $0.1,1$ or $10 \mu \mathrm{M}$ lenalidomide (Sigma) or $0.01 \%$ dimethyl sulfoxide control. Half the volume of media, with cytokines, was replaced weekly. At day 22, cells were harvested on ice, passed through a $50 \mu \mathrm{m}$ filter, washed and stained for flow cytometric analysis.

Statistics. Graphs were plotted with Prism V5 (GraphPad software Inc.) and mean, 95\% confidence interval, s.e.m., s.d., linear regression analysis and Mann-Whitney $U$-tests (two-tailed) were calculated within the software.

Data availability. The authors declare that the data supporting the findings of this study are available within the article and its Supplementary Information files, or are available upon reasonable requests to the authors.

Received: 11 July 2017 Accepted: 10 January 2018

Published online: 27 March 2018

\section{References}

1. Milner, J. D. \& Holland, S. M. The cup runneth over: lessons from the everexpanding pool of primary immunodeficiency diseases. Nat. Rev. Immunol. 13, 635-648 (2013)
2. Collin, M., Bigley, V., Haniffa, M. \& Hambleton, S. Human dendritic cell deficiency: the missing ID? Nat. Rev. Immunol. 11, 575-583 (2011).

3. Kapsenberg, M. L. Dendritic-cell control of pathogen-driven T-cell polarization. Nat. Rev. Immunol. 3, 984-993 (2003).

4. Sapoznikov, A. et al. Perivascular clusters of dendritic cells provide critical survival signals to B cells in bone marrow niches. Nat. Immunol. 9, 388-395 (2008).

5. Reboldi, A. et al. IgA production requires B cell interaction with subepithelial dendritic cells in Peyer's patches. Science 352, aaf4822 (2016).

6. Guimond, M. et al. In vivo role of Flt3 ligand and dendritic cells in NK cell homeostasis. J. Immunol. 184, 2769-2775 (2010).

7. Sallusto, F. et al. Distinct patterns and kinetics of chemokine production regulate dendritic cell function. Eur. J. Immunol. 29, 1617-1625 (1999).

8. Haniffa, M., Bigley, V. \& Collin, M. Human mononuclear phagocyte system reunited. Semin. Cell Dev. Biol. 41, 59-69 (2015).

9. Swiecki, M. \& Colonna, M. The multifaceted biology of plasmacytoid dendritic cells. Nat. Rev. Immunol. 15, 471-485 (2015).

10. Lauterbach, H. et al. Mouse CD8alpha+DCs and human BDCA3+DCs are major producers of IFN-lambda in response to poly IC. J. Exp. Med. 207, 2703-2717 (2010)

11. Nizzoli, G. et al. Human CD1c+dendritic cells secrete high levels of IL-12 and potently prime cytotoxic T-cell responses. Blood 122, 932-942 (2013).

12. Murphy, T. L. et al. Transcriptional control of dendritic cell development. Annu. Rev. Immunol. 34, 93-119 (2016).

13. Dickinson, R. E. et al. The evolution of cellular deficiency in GATA2 mutation. Blood 123, 863-874 (2014).

14. Cisse, B. et al. Transcription factor E2-2 is an essential and specific regulator of plasmacytoid dendritic cell development. Cell 135, 37-48 (2008).

15. Jaiswal, H. et al. Batf3 and Id 2 have a synergistic effect on Irf8-directed classical CD8alpha+dendritic cell development. J. Immunol. 191, 5993-6001 (2013).

16. Hambleton, S. et al. IRF8 mutations and human dendritic-cell immunodeficiency. N. Engl. J. Med. 365, 127-138 (2011).

17. Sichien, D. et al. IRF8 transcription factor controls survival and function of terminally differentiated conventional and plasmacytoid dendritic cells, respectively. Immunity 45, 626-640 (2016).

18. Schlitzer, A. et al. IRF4 transcription factor-dependent CD11b+dendritic cells in human and mouse control mucosal IL-17 cytokine responses. Immunity 38, 970-983 (2013).

19. Feinberg, M. W. et al. The Kruppel-like factor KLF4 is a critical regulator of monocyte differentiation. EMBO J. 26, 4138-4148 (2007).

20. Patel, A. A. et al. The fate and lifespan of human monocyte subsets in steady state and systemic inflammation. J. Exp. Med. 214, 1913-1923 (2017).

21. Merkenschlager, M. Ikaros in immune receptor signaling, lymphocyte differentiation, and function. FEBS Lett. 584, 4910-4914 (2010).

22. Allman, D. et al. Ikaros is required for plasmacytoid dendritic cell differentiation. Blood 108, 4025-4034 (2006).

23. Wu, L., Nichogiannopoulou, A., Shortman, K. \& Georgopoulos, K. Cellautonomous defects in dendritic cell populations of Ikaros mutant mice point to a developmental relationship with the lymphoid lineage. Immunity 7 , 483-492 (1997).

24. Lucioni, M. et al. Twenty-one cases of blastic plasmacytoid dendritic cell neoplasm: focus on biallelic locus 9p21.3 deletion. Blood 118, 4591-4594 (2011).

25. Kuehn, H. S. et al. Loss of B cells in patients with heterozygous mutations in IKAROS. N. Engl. J. Med. 374, 1032-1043 (2016).

26. Hoshino, A. et al. Abnormal hematopoiesis and autoimmunity in human subjects with germline IKZF1 mutations. J. Allergy Clin. Immunol.. 140, 223-231 (2016).

27. Bogaert, D. J. et al. A novel IKAROS haploinsufficiency kindred with unexpectedly late and variable B-cell maturation defects. J. Allergy Clin. Immunol. 141, 432-435.e7 (2017).

28. Krönke, J. et al. Lenalidomide causes selective degradation of IKZF1 and IKZF3 in multiple myeloma cells. Science 343, 301-305 (2014).

29. $\mathrm{Lu}, \mathrm{G}$. et al. The myeloma drug lenalidomide promotes the cereblon -dependent destruction of Ikaros proteins. Science 343, 305-309 (2014).

30. Van Gassen, S. et al. FlowSOM: Using self-organizing maps for visualization and interpretation of cytometry data. Cytom. A 87, 636-645 (2015).

31. European Medicines Agency. Assessment Report, Revlimid.Report No. EMEA/ H/C/000717/II/0056 (European Medicines Agency, 2013).

32. Abdulhay, N. et al. Normal hematologic parameters and fetal hemoglobin silencing with heterozygous IKZF1 mutations. Blood 128, 2100-2103 (2016).

33. Rouillard, A. D. et al. The harmonizome: A collection of processed datasets gathered to serve and mine knowledge about genes and proteins. Database (Oxford) http://amp.pharm.mssm.edu/Harmonizome/terms (2016).

34. Siedlar, M. et al. Preparations of intravenous immunoglobulins diminish the number and proinflammatory response of CD14+CD16++monocytes in 
common variable immunodeficiency (CVID) patients. Clin. Immunol. 139, 122-132 (2011).

35. Gamrekelashvili, J. et al. Regulation of monocyte cell fate by blood vessels mediated by Notch signalling. Nat. Commun. 7, 12597 (2016).

36. Witkowski, M. T. et al. Activated Notch counteracts Ikaros tumor suppression in mouse and human T-cell acute lymphoblastic leukemia. Leukemia $\mathbf{2 9}$, 1301-1311 (2015).

37. Gopalakrishnan, R., Matta, H., Tolani, B., Triche, T. \& Chaudhary, P. M. Immunomodulatory drugs target IKZF1-IRF4-MYC axis in primary effusion lymphoma in a cereblon-dependent manner and display synergistic cytotoxicity with BRD4 inhibitors. Oncogene 35, 1797-1810 (2016).

38. Vo, M. C. et al. Lenalidomide enhances the function of dendritic cells generated from patients with multiple myeloma. Exp. Hematol. 46, 48-55 (2017).

39. Costa, F. et al. Lenalidomide increases human dendritic cell maturation in multiple myeloma patients targeting monocyte differentiation and modulating mesenchymal stromal cell inhibitory properties.Oncotarget 8, 53053-53067 (2017).

40. Movassagh, M., Laderach, D. \& Galy, A. Proteins of the Ikaros family control dendritic cell maturation required to induce optimal Th1 $\mathrm{T}$ cell differentiation. Int. Immunol. 16, 867-875 (2004).

41. Sung, M. H. et al. Switching of the relative dominance between feedback mechanisms in lipopolysaccharide-induced NF-kB signaling. Sci. Signal. 7, ra6 (2014).

42. Baillie, J. K. et al. Analysis of the human monocyte-derived macrophage transcriptome and response to lipopolysaccharide provides new insights into genetic aetiology of inflammatory bowel disease. PLoS Genet. 13, e1006641 (2017).

43. Gautier, G. et al. A type I interferon autocrine-paracrine loop is involved in Toll-like receptor-induced interleukin-12p70 secretion by dendritic cells. J. Exp. Med. 201, 1435-1446 (2005).

44. Crother, T. R. et al. Plasmacytoid dendritic cells play a role for effective innate immune responses during Chlamydia pneumoniae infection in mice. PLoS ONE 7, e48655 (2012).

45. Poeck, $\mathrm{H}$. et al. Plasmacytoid dendritic cells, antigen, and CpG-C license human B cells for plasma cell differentiation and immunoglobulin production in the absence of T-cell help. Blood 103, 3058-3064 (2004).

46. Matta, B. M., Castellaneta, A. \& Thomson, A. W. Tolerogenic plasmacytoid DC. Eur. J. Immunol. 40, 2667-2676 (2010).

47. Chauhan, D. et al. Functional interaction of plasmacytoid dendritic cells with multiple myeloma cells: a therapeutic target. Cancer Cell 16, 309-323 (2009).

48. Nguyen-Pham, T. N. et al. Lenalidomide synergistically enhances the effect of dendritic cell vaccination in a model of murine multiple myeloma. $J$. Immunother. 38, 330-339 (2015).

49. Noonan, K. et al. Lenalidomide-induced immunomodulation in multiple myeloma: impact on vaccines and antitumor responses. Clin. Cancer Res. 18, 1426-1434 (2012).

50. Kim, J. M., Park, S. H., Kim, H. Y. \& Kwok, S. K. A plasmacytoid dendritic cells-type I interferon axis is critically implicated in the pathogenesis of systemic lupus erythematosus. Int J. Mol. Sci. 16, 14158-14170 (2015).

\section{Acknowledgements}

We gratefully acknowledge the participation of the patients and their families. We thank Rachel Queen from Newcastle University Single Cell Unit for bioinformatics support. We acknowledge the Newcastle University Flow Cytometry Core Facility (FCCF) for assistance with the generation of Flow Cytometry data. This study was funded by Wellcome Trust 101155/Z/13/Z (V.B., U.C.), CGD Society CGDS16/01, EU-FP7 CELL-PID HALTH-2010-261387, EU-FP7 NET4CGD (J.R.), Jeffrey Modell Foundation (S.P., F.H M.D.), Intramural research program of the NIH Clinical Center (H.S.K., S.D.R.), NIH R21 AI094004 and NIH R03 AI113631 (A.K., K.V.V., H.H.), Research Foundation Flanders (D.B.), Ghent University Hospital Spearhead Initiative for Immunology Research (D.B., M.D., F.H.).

\section{Author contributions}

U.C. conducted experiments, analysed results and wrote the manuscript; A.R. analysed results and wrote the manuscript; T.A. conducted experiments and analysed results; A.G. supervised experiments and appraised the manuscript; D.B., H.S.K., G.J., A.K., K.V.V., S. P., M.D., J.R., H.H. and F.H. provided patient material and appraised the manuscript. S. D.R. conceptualised the study, provided patient material and appraised the manuscript; M.C. conceptualised the study, designed experiments and wrote the manuscript; V.B. conceptualised the study, designed and conducted experiments, analysed results and wrote the manuscript.

\section{Additional information}

Supplementary Information accompanies this paper at https://doi.org/10.1038/s41467018-02977-8.

Competing interests: The authors declare no competing financial interests.

Reprints and permission information is available online at http://npg.nature.com/ reprintsandpermissions/

Publisher's note: Springer Nature remains neutral with regard to jurisdictional claims in published maps and institutional affiliations.

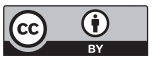

Open Access This article is licensed under a Creative Commons Attribution 4.0 International License, which permits use, sharing, adaptation, distribution and reproduction in any medium or format, as long as you give appropriate credit to the original author(s) and the source, provide a link to the Creative Commons license, and indicate if changes were made. The images or other third party material in this article are included in the article's Creative Commons license, unless indicated otherwise in a credit line to the material. If material is not included in the article's Creative Commons license and your intended use is not permitted by statutory regulation or exceeds the permitted use, you will need to obtain permission directly from the copyright holder. To view a copy of this license, visit http://creativecommons.org/ licenses/by/4.0/.

(c) The Author(s) 2018 